\title{
PIMREG expression level predicts glioblastoma patient survival and affects temozolomide resistance and DNA damage response
}

Rodolfo Bortolozo Serafim

Universidade de São Paulo (USP)

Cibele Cardoso

Universidade de São Paulo (USP)

Vanessa Arfelli

Universidade de São Paulo (USP)

Valeria Valente

Universidade Estadual Paulista (UNESP)

Leticia Fröhlich Archangelo ( $\sim$ leticiafa@fmrp.usp.br)

Universidade de São Paulo (USP)

\section{Research Article}

Keywords: PIMREG expression, glioblastoma, temozolomide, DNA damage response

Posted Date: March 19th, 2021

DOl: https://doi.org/10.21203/rs.3.rs-322224/v1

License: (c) (i) This work is licensed under a Creative Commons Attribution 4.0 International License. Read Full License

Version of Record: A version of this preprint was published at Biochimica et Biophysica Acta (BBA) Molecular Basis of Disease on March 1st, 2022. See the published version at https://doi.org/10.1016/j.bbadis.2022.166382. 


\section{Abstract}

PIMREG expression strongly correlates with cellular proliferation in both malignant and normal cells. Throughout embryo development, PIMREG expression is prominent at the central nervous system. Recent studies have described high levels of PIMREG transcripts in different types of tumors and correlated with patient survival and tumor aggressiveness. Given the emerging significance of PIMREG in carcinogenesis and its putative role in the context of the nervous system, we investigated the expression and function of PIMREG in gliomas, the most common primary brain tumors. We performed an extensive analysis of PIMREG expression in tumors samples of glioma patients, assessed the effects of PIMREG silencing and overexpression on the sensitivity of glioblastoma cell lines treated with genotoxic agents commonly used for treating patients and assessed for treatment response, proliferation and migration. We show that glioblastoma exhibits the highest levels of PIMREG expression among all cancers analyzed and that elevated PIMREG expression is a biomarker for glioma progression and patient outcome. Moreover, PIMREG is induced by genotoxic agents and its silencing renders glioblastoma cells sensitive to temozolomide treatment and affects ATR- and ATM-dependent signaling. Our data demonstrate that PIMREG plays a role in DNA damage response and temozolomide resistance of glioblastoma cells and further support the PIMREG role in tumorigenesis.

\section{Introduction}

Gliomas are the most common primary brain tumors ${ }^{1}$ and are classified according the World Health Organization (WHO) histological and grade (I-IV) criteria. The most common gliomas include astrocytomas (grade I-IV), oligodendroglioma (grade II-III) and oligoastrocytoma (grades II-III) ${ }^{2}$. Survival varies significantly by grade across glioma subtypes ${ }^{3}$. Glioblastoma (GBM) is the most aggressive and malignant subtype (astrocytomas of grade IV) and corresponds to more than $50 \%$ of all glioma cases. Patients with GBM have the poorest outcome, with an average survival of 15 months ${ }^{4}$. The standard care for primary GBMs comprises surgical resection and concurrent temozolomide (TMZ) with postoperative radiation. However, tumors invariably become resistant and recur ${ }^{5}$.

Recent studies on molecular profiling of gliomas have identified genetic, gene expression and DNA methylation signatures associated with prognosis ${ }^{6}$. These findings have helped to define molecular entities and improve classification and predict to treatment response $5,7,8$. Presently, biomarkers used in routine diagnostics are limited to Isocitrate dehydrogenase (IDH) mutations, deletions of the chromosomes 1p and 19q in oligodendroglial tumors and 0-6-Methylguanine-DNA Methyltransferase (MGMT) hypermethylation in GBM. Thus, it is crucial to identify novel biomarkers of prognostic value and for characterization of molecular mechanisms of radio- and chemoresistance.

The PICALM interacting mitotic regulator (PIMREG, also known as CATS, FAM64A and RCS1) is a marker for proliferation highly expressed in tumor cell lines ${ }^{9}$. PIMREG was shown to be regulated in a cell cycle dependent manner and to control the metaphase to anaphase transition in mitotic cells ${ }^{10}$. 
In normal adult tissue PIMREG expression is restricted to the thymus, spleen, and colon and, to a lesser extent, the small intestine, ovary, and brain ${ }^{9,11}$. During embryogenesis expression is widely distributed in the embryo, with marked expression in the central nervous system (CNS). Notably, the striking expression of this gene's activity in the neural tube in early stages of development decays abruptly at a specific stage of development ${ }^{9}$. The particular high expression of Pimreg in the developing CNS and the fact that it is precisely regulated in a temporal-spatial manner suggest that Pimreg could be particularly important for the development of this tissue.

First functional evidence that PIMREG plays a role in cellular processes involved in tumorigenesis was provided when silencing the gene resulted in reduced proliferation, altered cell cycle progression, and decreased expression of cell cycle regulators, as well as impaired migratory and clonogenic abilities of leukemia cells ${ }^{12}$.

Also, a variety of studies have described high levels of PIMREG transcripts in different types of tumors. In an integrated analysis of gene expression profiles of tumors samples from breast cancer patients, PIMREG was identified together with BIRC5 and CENPA, as the three-gene signature specifically upregulated in triple-negative breast cancer. In a broader clinical analysis, high expression of PIMREG correlates with poor survival, regardless the molecular type of breast cancer, drawing attention to its potential value as a prognostic biomarker ${ }^{13}$. In another study, a Pan-cancer analysis based on gene expression profiles of paired tumor and adjacent normal tissues, was carried out on 633 samples across 13 different types of cancer. The study identified high expression of PIMREG in a variety of cancer types and its expression correlated with poor patient survival ${ }^{14}$. Furthermore, a possible role of PIMREG as a marker of tumor progression and prognosis has been proposed for breast cancer ${ }^{13-17}$, renal cell carcinoma ${ }^{18,19}$ and pancreatic cancer ${ }^{20}$. Moreover, PIMREG was described as one of a four-gene signature predictive as biomarker for paclitaxel response in HER-2 negative breast cancers ${ }^{21}$. Further, parallel studies conducted by different groups showed that PIMREG is involved the migration, metastasis, epithelial-mesenchymal transition and aggressiveness of breast cancer ${ }^{15-17}$.

More recently, an important function of PIMREG in regulating inflammation and inflammation-dependent carcinogenesis was demonstrated. Xu and colleagues reported that Pimreg deleted mice exhibited impaired differentiation of Th17 lymphocytes, altered gene expression signature and pro-inflammatory activity characteristic of these cells. As a consequence, Pimreg appears to play a key role in the development of Th17-dependent inflammatory diseases in animal models, such as autoimmune encephalomyelitis, dextran sulfate sodium-induced colitis and colitis-associated carcinogenesis 22 . Pimreg regulates Th17 cell differentiation through interaction with the activated STAT3, retaining this transcription factor in the nucleus and thus contributing to the regulation of its target genes 22 .

The interaction of PIMREG with transcription regulators that shuttle between the nucleus and cytoplasm, and consequent retention of these factors in the nuclear compartment and modulation of its transcriptional activities, seem to be a recurrent mode of action of PIMREG. We have previously shown 
that through PICALM interaction PIMREG increases the nuclear localization of the leukemic fusion protein PICALM/MTT10 (CALM/F10) and interferes with its ability to activate transcription activate transcription in a dose-dependent manner ${ }^{11,23}$.

A similar mechanism was also described for a PIMREG-mediated regulation of NFkB signaling pathway in breast cancer cells ${ }^{15}$. In that report the authors showed that PIMREG promotes the aggressiveness of breast cancer by constitutively activating the NF-kB pathway. PIMREG interacts with the p65 protein at the same binding site as its inhibitor IkB, thus competing for the interaction site. In cells that express high levels of PIMREG, such as breast cancer cells, interaction with p65 occurs in the nucleus, which leads to stabilization of this transcription factor in this compartment, binding to its responsive elements and consequent activation of its target genes ${ }^{15}$.

Given the emerging significance of PIMREG in carcinogenesis and its putative role in the context of nervous system, we sought to investigate the expression and function of PIMREG in gliomas. Here we show that patients with most aggressive and malignant glioma subtype, namely GBM, exhibit the highest levels of PIMREG expression among all cancers analyzed. PIMREG expression correlates with glioma progression and impacts patient overall survival. We performed an extensive expression and function analyzes of PIMREG in GBM cell lines treated with genotoxic agents commonly used for treating GBM patients and assessed for treatment response, proliferation and migration. We show that PIMREG is induced by genotoxic agents and its silencing renders GBM cells more sensitive to TMZ treatment and affects DNA damage signaling. Our data suggest that PIMREG plays a role in DNA damage response and TMZ resistance of GBM cells.

\section{Results}

\section{PIMREG is overexpressed in GBM and its expression levels correlates with tumor progression and patient survival}

We performed a comparative analysis of PIMREG mRNA levels in paired tumor $(\mathrm{n}=9475)$ and adjacent non-tumor tissues ( $n=773$ ) across 25 different types of cancer from the TCGA dataset. The analysis confirmed that PIMREG is highly expressed across all tumor types in comparison to the normal tissue. Among all tumors analyzed, GBM showed the highest levels of PIMREG expression (Fig. 1A). Interestingly, the median expression of PIMREG in GBM patient samples was 8.932 RSEM (log2) while in the combined cohort that included lower-grade gliomas (GBMLGG), it was 4.99 RSEM (log2), indicating that lower-grade gliomas (LGG) express lower levels of PIMREG in comparison to the more aggressive subtype, GBM. To test this hypothesis, we evaluated the PIMREG expression in the GBMLGG group $(n=660)$ classified according to WHO grades (II, III and IV), which in turn reflect the stages of malignancy.

PIMREG lowest expression was observed in patients with grade II gliomas, which is comprised by astrocytoma, oligodendroglioma and oligoastrocytoma samples and exhibit the least malignant phenotype among the three groups examined. Intermediate levels of PIMREG expression were observed in 
patients with grade III gliomas, which consists of anaplastic oligoastrocytoma and anaplastic astrocytoma and present a more aggressive phenotype compared to grade II gliomas. Finally, highest levels of PIMREG were observed in patients with grade IV gliomas or GBM, which is the group with the most aggressive phenotype (Fig. 1B). Thus, PIMREG expression positively correlates with the degree of malignancy in glioma with the highest expression observed in the subgroup with the most malignant phenotype.

It is well accepted that low grade gliomas carrying mutations in the Isocitrate dehydrogenase 1 (IDH1) gene have a better prognosis in comparison to gliomas with the wild type gene ${ }^{24}$. Thus, we evaluated the PIMREG expression in the LGG patient tumors classified according IDH1 status. PIMREG expression was significantly lower in tumors carrying IDH1 mutation (IDH1 mut) than tumors carrying the wild-type gene (IDH1wt) (Fig. 1C). Similarly, lowers levels of PIMREG were observed in LGG tumors carrying mutations in the homolog of Drosophila capicua gene (CIC) (Fig. S1). CIC mutations are characteristic of oligodendrogliomas that harbor co-deletion of chromosome $1 \mathrm{p}$ and $19 \mathrm{p}^{25}$, which is also a predictor of improved survival ${ }^{26}$.

In order to analyze the impact of PIMREG expression on patient outcome, we analyzed the survival curve of the patients from GBMLGG-TCGA dataset $(n=660)$ and three additional cohorts from published studies ${ }^{27-29}$. Kaplan-Meier curves showed that high levels of PIMREG negatively impact the survival of glioma patients (Fig. 1D). In the GBMLGG -TCGA cohort $(n=660)$, the median survival for the group of patients with high PIMREG expression was 742 days, while for the group with low PIMREG expression was 2907 days. Similar pattern was observed for the validation cohorts. In the study by Freije et al. (GSE4412) ${ }^{27}$, the median survival for glioma patients with high PIMREG expression was 223 days whereas for the patients with low expression it was 726 days, in the study by Gravendeel et al., $\left(\right.$ GSE16011) ${ }^{28}$ the median survival for the group of patients with high PIMREG expression was 11.28 months, and with low expression was 26.52 months. Finally, in the study by Phillips et al., (GSE4271) ${ }^{29}$ the median survival for the group of patients with high PIMREG expression was 17.5 months, and for the group with low expression it was 28 months. The data presented demonstrates that PIMREG expression correlates with glioma progression, and patients with high expression of this gene in their tumors have worse prognosis than those with low expression.

\section{PIMREG expression is co-regulated with genes enriched for cell cycle, DNA replication and repair pathways}

In order to identify cellular processes in which PIMREG may play a role in malignant glioma, we performed a pathway enrichment analysis based on the genes whose expression co-regulated with PIMREG in GBM patient samples (GBM - provisional TCGA $\mathrm{n}=604)$. The genes which presented a positive or negative correlation with PIMREG (Sperman's correlation coefficient ${ }^{3} 0.51$ and $^{3}-0.51$ ) were selected and submitted to pathway enrichment analysis through the DAVID database (https://david.ncifcrf.gov/home.jsp). The first 10 key pathways with statistical power were enriched for cell cycle function, DNA replication, pyrimidine metabolism and DNA repair pathways, such as Fanconi 
anemia pathway, mismatch repair (MMR), homologous recombination (HR) and nucleotide excision repair (NER) (Fig. 2A). The list of genes in which expression covaries with that of PIMREG in each pathway is provided in Table S1.

\section{PIMREG is highly expressed in GBM cell lines}

Next, we analyzed PIMREG gene and protein expression in GBM, LGG and the non-tumor astrocytes (ACBRI371) cell lines. With the exception of U87MG cells, PIMREG mRNA levels were as high as 2 to 3fold in GBM cell lines (SF188, T98G, U138MG, U251MG and U343MG) when compared to ACBRI371 and LGG cells (Fig. 2B). Similarly, protein levels were higher in the GBM cell lines SF188, T98G, U251MG and U343MG, although lower levels were observed in U87MG and U138MG cells (Fig. 2C).

\section{PIMREG is upregulated in GBM cells treated with genotoxic agents}

PIMREG protein expression was evaluated in GBM U87MG and T98G cells treated with genotoxic agents (TMZ, cisplatin and hydroxyurea) and ionizing radiation (IR), commonly used to treat cancer. PIMREG is localized to the nucleoplasm and nuclear expression levels increased progressively in U87MG and T98G cells treated with increasing concentrations of TMZ, reaching up to 3-fold expression at the concentration of $400 \mu \mathrm{M}$ of the drug. The dose-dependent increase in PIMREG expression was accompanied by phosphorylation of the histone $\mathrm{H} 2 \mathrm{AX}$ at serine 139 (gH2AX), used as a marker for DNA damage (Fig. 3A). Similar results were observed when U87MG cells were treated with increasing amounts of hydroxyurea and cisplatin (Fig.S2A). PIMREG is known to be a marker for proliferation enriched at G2/M phases of the cell cycle ${ }^{9}$. Thus, we performed a cell cycle analysis in order to investigate whether PIMREG upregulation in TMZ-treated cells was a consequence of altered cell cycle progression. The cell distribution along the different phases of the cell cycle were similar for TMZ-treated and non-treated cells, demonstrating that cell cycle was not affected by the TMZ treatment (Fig. 3B). Hence, PIMREG upregulation was not due to increased proliferation of the GBM treated cells nor its arrest at G2 phase.

Exposure of U87MG and T98G to increasing doses of IR also induced accumulation of PIMREG protein, which reached up to 1.7-fold at higher exposure doses (3-7 Gy) in both cell lines (Fig. 3C). Cell cycle analysis demonstrated that IR treatment resulted G2/M phase arrest in both cell lines (Fig. 3D).

In addition, we evaluated the fluorescence intensity of PIMREG protein in U87MG cells before and after TMZ treatment, together with the expression of Cyclin A, a cell cycle regulator whose expression during S/G2 phases of the cell cycle mirrors that of PIMREG ${ }^{9}$. The immunofluorescence analysis confirmed PIMREG upregulation in TMZ-treated cells. Moreover, PIMREG protein increase was restricted to Cyclin A positive cells, whereas cells that do not have Cyclin A labeling showed no upregulation of PIMREG. Of note, TMZ treatment did not alter the levels of Cyclin A protein (Fig. 3E). These results indicate that PIMREG is upregulated in TMZ-treated cells restricted to S/G2 phases of the cell cycle. Furthermore, marked upregulation of PIMREG was observed in U87MG cells treated with cisplatin and hydroxyurea. In these cells, the enhanced PIMREG expression was observed throughout the nucleoplasm and did not assemble at DNA damage foci, as assessed by yH2AX staining (Fig. S2B). 


\section{PIMREG knockdown sensitizes GBM cells to TMZ treatment}

To better understand the PIMREG role in GBM cells, we generated U87MG and T98G cells carrying constructs for PIMREG knockdown and overexpression. The sequence named as miPIMREG\#1 showed $74 \%$ and $91 \%$ reduction of the PIMREG protein in U87MG and T98G cells, respectively. The miPIMREG\#2 sequence was not effective to deplete PIMREG in either cell lines and was therefore used as negative control in the experiments to follow. The overexpression vector (PIMREG) led to an increase of PIMREG protein levels by 634- and 317-fold in U87MG and T98G cells, respectively (Fig. 4A and Fig. S3A). Sensitivity towards TMZ treatment was assessed in PIMREG depleted and overexpressing U87MG and T98G cells.

Clonogenic survival assay demonstrated that GBM cells depleted of PIMREG (miPIMREG\#1) formed less colonies than control cells at different concentrations of TMZ treatment (Fig. 4B). The PIMREG overexpression did not impact TMZ sensitivity in either cell lines at the indicated concentrations of treatment (Fig. S4). These results indicate that PIMREG silencing renders GBM cells more responsive to TMZ treatment.

\section{PIMREG knockdown impairs ATM activation and ATR signaling in TMZ-treated GBM cells}

In order to investigate a possible relationship between PIMREG and DNA damage response, we evaluated the initial DNA damage signaling cascade in PIMREG depleted and overexpressing U87MG and T98G cells treated with TMZ.

DNA damage response was assessed by Western blotting analysis using antibodies against the activated ATM (phospho-ATM-S1981) and ATR (phospho-ATR-S428) apical sensor kinases of DNA damage [reviewed in 42] and the replication protein A subunit 32 (phospho-RPA32-S33). Phosphorylation of ATM (S1981) was markedly impaired in PIMREG depleted U87MG and T98G cells subjected to TMZ treatment. The reduction of phosphorylation levels was $73 \%$ and $53 \%$ in the U87MG and T98G cells, respectively, when compared to miLUC control cells. PIMREG overexpression did not impact ATM phosphorylation at the presented condition (Fig. 5A). Additionally, phosphorylation of ATR was not altered by PIMREG depletion or overexpression (Fig. 5B). However, phosphorylation of ATR target RPA32(S33), was reduced in PIMREG depleted U87MG cells subjected to TMZ treatment (Fig. 5C). These results demonstrate that PIMREG knockdown impairs ATM activation as well as ATR-dependent downstream signaling in response to TMZ-induced DNA damage.

\section{TMZ-mediated upregulation of PIMREG is dependent on ATR activity}

Next, we analyzed the ability of TMZ treatment to induce PIMREG expression in U87MG cells impaired of DNA damage response pathways. As previously demonstrated, U87MG cells proficient for both ATM and ATR pathways exhibited increased PIMREG expression upon TMZ treatment in comparison to non-treated parental cells (Fig. 3A). In contrast, TMZ treatment was unable to upregulate PIMREG expression in U87MG cells inhibited for ATR pathway, where PIMREG levels remained comparable to non-treated cells 
(Fig. 5D). Also, ATM inhibition slightly enhanced the PIMREG upregulation elicited by TMZ treatment. Although this difference was not statistically significant, it was a consistently observed. These results demonstrated that PIMREG is a downstream target of ATR regulation and further confirmed a PIMREG/ATM regulatory axis.

\section{PIMREG knockdown reduces proliferation of GBM cells}

We further investigated PIMREG function on proliferation of GMB cells. PIMREG silenced or overexpressing GBM cells were assessed for cell growth on day $0,1,3,5,7$ and 9 of culture with FBSdepleted medium. Cell number was markedly reduced in PIMREG silenced T98G cells as detected after 5 days of culture, reaching $40 \%$ the number of the miLUC control cells after 9 days of growth (Fig. 6A). Hampered cell cycle progression was further confirmed by the decreased expression of Cyclin $A$ and Cyclin B in PIMREG silenced T98G cells (Fig. 6B). Proliferation rate was not affected in T98G cells ectopically expressing PIMREG. Likewise, U87MG cell proliferation was not affected upon modulation of PIMREG expression (Fig. S5).

\section{PIMREG knockdown reduces migration of GBM cells}

We next investigated the impact of PIMREG expression on migration of GBM cells. Cell migration was assessed in PIMREG silenced and overexpressing cell by wound healing and transwell migration assays. The wound healing assay demonstrated that PIMREG silenced T98G cells exhibited decreased rate of wound closure of $25 \%$ and $36 \%$ at 72 and 144 hours after the scratch, respectively. In control cells the wound was fully closed at 144 hours after scratching, whereas PIMREG silenced cells exhibited $50 \%$ of the wound unrepaired at this time point (Fig. 7A). Annexin V / PI analysis of PIMREG silenced and control T98G cells at 144 hours after scratch showed no differences in viability or cell death (Fig. S6A). Thus, the decreased rate of wound healing by PIMREG silenced T98G cells was not due to cell death but rather impaired migration of these cells.

Next, we analyzed the impact of PIMREG expression on migration towards a chemotaxis stimulus. In transwell migration assays PIMREG depleted U87MG and T98G cells migrated $26 \%$ and $29 \%$ less than miLUC control cells (Fig. 7B).

PIMREG ectopic expression did not impact migration rate of U87MG and T98G cells in the presented condition in both assays (Fig. S6B and S6C).

\section{Discussion}

In order to explore PIMREG function in primary brain tumors, such as gliomas, we performed an extensive analysis of PIMREG expression in tumors samples of glioma patients from the TCGA dataset and three additional independent cohorts, assessed the effects of PIMREG silencing and overexpression on the sensitivity of GBM cells to TMZ treatment and explored possible functions of PIMREG in gliomagenesis and treatment response. 
PIMREG is a marker for proliferation ${ }^{9}$, and in keeping with this activity, it was found highly expressed in tumor samples compared to their respective normal tissue counterpart. GBM exhibited the highest levels of PIMREG expression among the 25 cancer types examined. It was previously shown that Pimreg is highly expressed in the CNS of developing embryos ${ }^{9}$, suggesting a particular role of this protein in the development of normal CNS. In line with this idea is the observation that the glioma-associated oncogene homolog 1 (GL/1) expression was down-regulated upon PIMREG silencing ${ }^{31}$. GLI1 is the positive effector of the Sonic-hedgehog signaling pathway, which is a key pathway in developing CNS ${ }^{32}$. Moreover, Gli1 plays an essential role in $\mathrm{GBM}^{33}$.

Expression analysis on patient samples showed that PIMREG mRNA levels correlate with glioma progression. Lower PIMREG transcript levels were consistently observed in well-defined subgroups characterized by favorable outcome, such as the mutated IDH and $\mathrm{CIC}$ subgroups. Moreover, survival curves dichotomized the group of patients with low versus high levels of PIMREG expression, the second exhibiting the shorter overall survival in all cohorts examined. These results suggest that elevated PIMREG expression could be a potential biomarker for tumor progression and predictive of patient outcome.

Pathway enrichment analysis in GBM patient samples revealed a strong association between PIMREG expression and that of genes enriched for cell cycle function, DNA replication and DNA repair pathways. The association of PIMREG and genes enriched for cell cycle function was foreseeable since PIMREG functions in proliferation ${ }^{9,34,35}$, cell cycle control ${ }^{31}$ and metaphase-to-anaphase transition ${ }^{10}$ were already reported. However, its putative involvement in a variety of DNA repair pathways was unexpected, and has not been previously described.

DNA repair pathways represent essential mechanisms of GBM cell resistance towards treatment and directly impact clinical outcome ${ }^{36}$. Thus, we explored the putative role of PIMREG in these processes and the possibility of PIMREG contributing to boost malignant phenotype in gliomas.

Treatment of GBM cells with various DNA damage-inducing agents triggered immediate increase of PIMREG protein, as cells accumulated DNA lesions. For TMZ-treated cells, the PIMREG protein accumulated in those cells undergoing $S$ and $G 2$ phases of the cell cycle. The accumulation was not a consequence of cell arrest in the phases where PIMREG is known to be more abundant, such as the $S$, G2/M phases of the cell cycle ${ }^{9,10}$, but most likely because PIMREG was required to help the cells to cope with the DNA insults elicited by the treatment. In contrast, IR treatment elicited cell arrest and thus, PIMREG accumulation was more likely to be due to the increased number of cells in the $G 2$ phase at the indicated dose.

GBM cells depleted of PIMREG were more sensitive to TMZ treatment than control cells, demonstrating that higher levels of PIMREG are necessary for the cells to better cope with the insults elicited by the TMZ treatment. Intriguingly, PIMREG overexpression was not able to increase the resistance of the GBM cells against TMZ. Since the GBM cell lines express a markedly high amount of PIMREG, it is likely that 
overexpression of exogenous PIMREG would not make a physiological appreciable difference as observed in PIMREG depleted cells at the indicated TMZ concentration.

Importantly, TMZ-treated GBM cells depleted of PIMREG presented impaired activation of the apical DNA damage response kinase ATM but not ATR. Although activation of ATR was not affected, the phosphorylation of its downstream target RPA32(S33) was reduced. RPA32-S33 is exclusively phosphorylated by ATR in response to a variety of replicative stress insults, necessary to activate S-phase checkpoint ${ }^{37-39}$, Thus, PIMREG may act downstream of ATR as an ATR-dependent signaling response to TMZ-induced DNA damage. Supporting this idea, GBM cells inhibited for ATR pathway were unable to upregulate PIMREG following TMZ treatment, whereas ATM inhibition slightly enhanced the PIMREG upregulation. These findings, not only demonstrate that PIMREG participate in the cellular response to DNA damage but also suggest the protein is a novel player in the signaling orchestrated by the sensor kinases ATM and ATR. We propose that PIMREG may act upstream of ATM and downstream of ATR, where it may facilitate ATR-dependent RPA32 (S33) phosphorylation.

Classically, ATM is master regulator of cellular responses to DSBs whereas ATR is responsible for regulating DNA replication stress response. Also, both ATM and ATR contribute to establishment and maintenance of the intra-S and G2/M checkpoints and there is considerable crosstalk between their pathways ${ }^{30}$. An example of functional interaction between these enzymes, is ATM-dependent DNA-end resection provides the RPA-ssDNA signal for ATR recruitment and activation ${ }^{40}$. Also, ATR function upstream of ATM in the response to UV or replication fork stall ${ }^{41}$. Whether PIMREG participates in separate ATM- and ATR-mediated signaling pathways or if it takes part in the crosstalk between these pathways remains to be further investigated. Of note, the serine residue at position 137 of PIMREG (S137) lies within a consensus site for ATM/ATR phosphorylation ${ }^{30}$. Indeed, the S137 of PIMREG was identified as a phosphosite in 3 different proteomic studies enriched for phospho-ATM/ATR substrates (PhosphoSitePlus ${ }^{\circledR}$ database (http://www.phosphosite.org). These findings further support PIMREG involvement in the ATM- and ATR-mediated signaling pathways.

We did not assess the precise nature of the damage caused by the treatments used in this study. Whether it caused replication stress with stalled replication forks, ssDNA, or dsDNA damage, and which of these mechanisms were responsible for causing PIMREG upregulation is not known at the present moment.

It is well known that TMZ methylates DNA at different positions and produces adducts to DNA bases. The most frequent lesions (N3-meG and N3-meA) are reversed by base excision repair (BER), whereas the less frequent and more cytotoxic 06-meG lesions are reversed by the direct repair mechanism. When removal of the 06-meG lesion is not effective, the presence of these alkylated bases causes mispairing during replication and consequent activation of the MMR ${ }^{42}$. This mechanism, in turn, does not restore 06-meG lesions, which can lead to the induction of single-strand breaks (SSBs) that, during DNA replication, are converted into double-strand breaks (DSBs) ${ }^{36}$. ATR is activated upon the presence of DNA lesions and replicative stress caused by TMZ treatment. In the proposed model, unsolved lesions and persistent stress would in turn lead to secondary lesions, such as ssDNA breaks and DSBs and 
consequently ATM activation ${ }^{43}$. It is conceivable to think that activated ATR regulates PIMREG, which in turn enhances ATR-downstream signaling, such as RPA phosphorylation and ATM activation.

We demonstrated that shRNA-mediated PIMEG silencing reduced cell proliferation up to $40 \%$ in the T98G GBM cells used in this study. Correspondingly, the expression of the cell cycle regulators cyclin A and cyclin B1 were also diminished in these cells. These results are consistent with our previous report in leukemia cells ${ }^{31}$, although in that study the reduction of proliferation upon PIMREG silencing was much milder compared to the observation using GBM cells in this study. Interestingly, depletion of PIMREG did not alter the proliferation of the other GBM cells analyzed (U87MG), indicating complex regulation must operate to control proliferative PIMREG function in other cellular contexts.

Given the PIMREG function on proliferation and cell-cycle control 9,10,31,34,35, together with the findings presented in this study that PIMREG participates in the DNA damage response with ATM and ATR, it is tempting to speculate that PIMREG may play a role in the ATR- and ATM-dependent checkpoint pathways to block cell cycle progression.

Finally, we demonstrated by two independent methods that PIMREG silencing reduces the migratory ability of GBM cells, confirming what was previous described for leukemia cells ${ }^{31}$. However, it is intriguing that PIMREG overexpression did not impact proliferation, ATM activation or cellular migration. One plausible explanation could be that the overexpression of the ectopic protein (634- and 317-fold over the endogenous expression in T98G and U87MG cells, respectively), would augment the levels of the protein beyond a threshold in which experimental variation would no longer be detected by the cell nor elicit a response. Further experiments assessing these questions in a more physiological context should be addressed.

Collectively, our data demonstrate for the first time that PIMREG plays a role in DNA damage response and TMZ resistance of GBM cells and further support the PIMREG role in the cellular processes involved in tumorigenesis.

\section{Materials And Methods}

\section{Public data analysis}

PIMREG mRNA expression data in primary solid tumors (TP) and adjacent normal tissues (NT) were downloaded from The Cancer Genome Atlas (TCGA) database using the Firebrowse portal ${ }^{44}$. Glioma patient clinical information, RNAseq-based mRNA expression and mutation data were downloaded from TCGA using cBioPortal for Cancer Genomics ${ }^{45}$. The datasets used for the analysis were Glioblastoma Multiform (TCGA, Firehose Legacy, previously known as provisional 604 samples) and Brain Lower Grade Glioma (TCGA, Firehose Legacy, previously known as provisional 530 samples) downloaded as October 2017 and TCGA-GBMLGG merged cohort ${ }^{6}$. PIMREG expression was analyzed in glioma patients stratified according to WHO grades (II, III and IV) and mutational status of recurrently affected genes. For survival 
analysis, expression data was retrieved through SurvExpress platform ${ }^{46}$, from GBMLGG (TCGA Gliomas - June $2016 n=660$ ) and three additional microarray-based datasets, GEO accession: GSE4412 27 , GSE16011 28 and GSE4271 29 .

Gene ontology was assessed with DAVID ${ }^{47}$ and pathway enrichment with KEGG. Graphs were plotted with GraphPad Prism 4.0 software.

\section{Glioma cell lines}

The GBM cell lines U87MG, U138MG, U251MG and T98G were obtained from the American Type Culture Collection (ATCC, Manassas, USA). The GBM explant derivative U343MG cells were obtained from Dr. Carlos Gilberto Carlotti Junior's laboratory (FMRP-USP). SF188 GBM cells and the lower-grade astrocytoma cells R186 (grade I), R259 (grade I) R286 (grade I) and UW467 (grade III) were provided by Dr. Michael Bobola (Washington University). The non-tumor astrocytes ACBRI371 were from Cell Systems (Cat\# ACBRI-371) ${ }^{48}$ and kindly provided by Dr. Elza Tiemi Sakamoto Hojo (FFCLRP-USP). All cell lines were grown in Dulbecco's Modified Eagle Medium supplemented with $10 \%$ fetal bovine serum (FBS) and incubated at $37^{\circ} \mathrm{C}$ and $5 \% \mathrm{CO}_{2}$.

All cell lines were tested for mycoplasm with the MycoAlert PLUS Mycoplasma Detection Kit (Lonza). U87MG and T98G were authenticated by STR matching analysis using the PowerPlex ${ }^{\circledR} 16$ HS system (Promega, Madison, WI, USA) and the ABI 3500 Sequence Detector System (Applied Biosystems, Foster City, CA, USA).

\section{Plasmid construction}

PIMREG HA-tagged cDNA was excised with Sfil restriction enzyme from the pSfiExp-CATS-HA construct ${ }^{23}$ and subcloned into the Doxycycline-inducible (Dox) episomal vector pRTS-1 (svh) ${ }^{49}$, digested with the same enzyme.

For conditional PIMREG knockdown, siRNA sequences targeting the 3' untranslated region (UTR) of the endogenous PIMREG mRNA embedded in the modified murine miR-155 structure were cloned into the Bpil site, downstream the eGFP open reading frame (ORF) of the subcloning pMIRTOP vector ${ }^{50}$. A fragment with the miRNA-embedded siRNA sequences (miPIMREG\#1: 5'-

tgctgaatagaaggctcaaggtcaaggttttggccactgactgaccttgacctagccttctatt-3' and miPIMREG\#2: 5'tgctgataaataggtacccgtgagccgttttggccactgactgacggctcacgtacctatttat-3') and the eGFP ORF were excised from the PMIRTOP plasmids with ECO47/I and $B g / / /$ restriction enzymes and the insert was subcloned into the BgIII and Swal digested pRTS-1 (svp) vector. pRTS constructs expressing luciferase ORF (pRTS-LUC) and the miRNA-embedded siRNA targeting luciferase (pRTS-miLUC), used as controls were previously described ${ }^{50}$.

The generated pRTS-PIMREG construct contains the hygromycin B resistance gene (hyg), whereas the pRTS-miPIMREG\#1 and -miPIMREG\#2 contains the puromycin gene for selection in mammalian cells. 
For a detailed description of the constructs contact the authors.

\section{Transfection of U87MG and T98G cells}

$1 \times 10^{5}$ U87MG and T98G cells were seeded at 6 -well plates and transfected with $5 \mu \mathrm{g}$ of plasmid DNA, Lipofectamine 3000 and P3000 reagent according to manufactures' instructions (Thermofisher). Stably transfected cell cultures were generated within approximately 7 days by hygromycin-B $(200 \mu \mathrm{g} / \mathrm{mL})$ and puromycin $(1 \mu \mathrm{g} / \mathrm{ml})$ selection of the overexpressing (pRTS-PIMREG and pRTS-LUC) and knockdown (pRTS-miPIMREG\#1, pRTS-miPIMREG\#2 and pRTS-miLUC) constructs, respectively. Cell populations were treated with doxycycline $(0,5 \mu \mathrm{g} / \mathrm{mL})$ for 2 days for conditional expression of the ectopic gene (PIMREG and LUC) or miRNA-embedded siRNA sequences (miPIMREG\#1, miPIMREG\#2 and miLUC).

\section{Cell treatment with genotoxic agents and kinase inhibitor}

Treatment with TMZ was carried out for 24 hours at concentrations of 25, 50, 100, 200, 400 and $600 \mu \mathrm{M}$ for western blot analysis and at $400 \mu \mathrm{M}$ for cell cycle and immunofluorescence experiments. For clonogenic assay, the cells were previously treated with $6.25,12.5,25$ and $50 \mu \mathrm{M} \mathrm{TMZ}$ for 24 hours. IR treatment was performed at doses of 1, 2, 3, 4 and $7 \mathrm{~Gy}$ for western blot analysis and at $4 \mathrm{~Gy}$ for cell cycle assays. Cells were treated with hydroxyurea for 24 hours at concentrations of $3.75,7.5,15,30$ and $60 \mu \mathrm{g} / \mathrm{mL}$ for western blot analysis and at $15 \mu \mathrm{g} / \mathrm{mL}$ for immunofluorescence assay. Cells were treated with cisplatin for 24 hours at concentrations of $0.25,0.5,1,2$ and $4 \mu \mathrm{g} / \mathrm{mL}$ for western blot analysis and at $1 \mu \mathrm{g} / \mathrm{mL}$ for immunofluorescence assay. For inhibition of ATM and ATR pathways cells were treated for 3 hours with $10 \mu \mathrm{M}$ of ATM (KU55933) and ATR (VE-821) kinase inhibitors prior to $400 \mu \mathrm{M} \mathrm{TMZ}$ treatment for 24 hours and cell lysis.

\section{Quantitative PCR}

Total RNA was isolated using the RNeasy RNA extraction Kit (Qiagen) following the manufacturer's instructions. DNAse I treated RNA was reverse transcribed with random primers and High Capacity Kit (Applied Biosystems). Reactions were carried out with Power SYBR Green PCR Master Mix, according to the manufactures' instructions (Applied Biosystems). The plates were run and analyzed by 7500 RealTime PCR System (Applied Biosystems). Primer sequences are available upon request. HPRT was used as endogenous control and relative gene expression was calculated using the $2^{-\triangle \Delta C T}$ equation ${ }^{51}$. Statistical analyzes were performed with the One-way ANOVA test, using the GraphPad Prism 4.0 software.

\section{Immunobloting and immunofluorescence}

Cells were lysed in RIPA buffer with protease inhibitor PMSF. Protein extracts were separated by electrophoresis on 6 or $12 \%$ SDS-polyacrylamide gel and transferred to a nitrocellulose membrane (Hybon-ECL; Amersham Biosciences, Arlington, IL). The membranes were blocked and probed with specific antibody, followed by incubation with secondary antibody conjugated to horseradish peroxidase 
(HRP). Protein signals were detected by chemiluminescence and the images were captured by ChemiDoc $^{\text {TM }}$ Imaging System (Bio-Rad). Primary antibodies were: anti-PIMREG (anti-CATS 2C4 (1:10) [9], anti- $\beta$-Actin (Cell Signaling, \#3700) (1:1000), anti-phospho-H2AX(S139) ( $\mathrm{H} 2 \mathrm{AX} ; \mathrm{Abcam}, \mathrm{ab} 26350)$ (1:1000), anti-phospho-ATM(S1981) (Abcam, ab81292) (1:1000), anti-ATM (Abcam, ab32420) (1:1000), anti-phospho-ATR(S428) (Cell Signaling, \#2853) (1:1000), anti-ATR (Cell Signaling, \#2790) (1:1000), antiphospho-RPA32/RPA2 (S33) (Abcam, ab87278) (1:200), anti-RPA32/RPA2 (Abcam, ab2175) (1:200), antiCyclin A (Santa Cruz, sc-271645) (1:500) and anti-Cyclin B (Santa Cruz, sc-7393) (1:500).

Secondary antibodies were purchased from Cell Signaling: anti-mouse (\#7076) (1:5000), anti-rat (\#7077) (1:2000) and anti-rabbit (\#7074) (1:5000). Densitometric quantification of the protein bands was performed with ImageJ v1.38. Statistical analyzes were performed with the One-way ANOVA test, using the GraphPad Prism 4.0 software.

For immunofluorescence, U87MG cells were grown on coverslips and treated with genotoxic agents for 24 hours. After that cells were fixed with $4 \%$ paraformaldehyde (PFA) for 15 minutes, permeabilized with PBS/0.25\% Triton X-100 for 15 minutes. Unspecific binding was blocked with PBS/5\% BSA for 1 hour. Coverslips were incubated with anti-PIMREG (CATS 2C4) mAb diluted 1:2, anti-Cyclin A (sc-271645) diluted 1:200 and anti-phospho-H2AX(S139) (Merck, \#05-636) diluted 1:500 at $4^{\circ} \mathrm{C}$ overnight in a humidified chamber. Secondary Alexa Fluor 488 (ThermoFisher, A-11006) and Alexa Fluor 594 (ThermoFisher, A-11005) antibodies diluted 1:500, were used for detection of the primary antibodies for 1 hour at RT. Nuclei were counterstained with DAPI (ThermoFisher, 62247) and cells were mounted on slides using ProLong ${ }^{\text {TM }}$ Gold Antifade Mountant (ThermoFisher, P10144). Images were captured by Leica DMI6000B fluorescence microscope. Statistical analyzes were performed with the Student's T-test, using the GraphPad Prism 4.0 software.

\section{Cell cycle analysis}

Cell cycle analysis was performed by DNA content quantification. Cells were washed with phosphatebuffered saline (PBS) with $5 \mathrm{mM}$ EDTA, fixed in 70\% ethanol for 30 minutes on ice, washed with PBSEDTA and stained with $20 \mu \mathrm{g} / \mathrm{mL}$ propidium iodide (PI) containing $10 \mu \mathrm{g} / \mathrm{mL}$ RNAse A for 30 minutes at $37^{\circ} \mathrm{C}$. Cell cycle was analyzed using FACSCanto (BD Biosciences) and ModFit LT 3.3 software. Statistical analyzes were performed with the Student's T-test, using the GraphPad Prism 4.0 software.

\section{Proliferation assay}

Stably transfected U87MG and T98G cells expressing of the ectopic gene (PIMREG and LUC) or miRNAembedded siRNA sequences (miPIMREG\#1, miPIMREG\#2 and miLUC) were seeded in 96-well plates at density of 500 cells/well. At the indicated time points, cells were washed, fixed in $70 \%$ ethanol for 10 minutes and stained with $0.5 \%$ crystal violet solution for 15 minutes. After that, cells were washed and incubated with $10 \%$ acetic acid solution for 30 minutes. Proliferation was evaluated by measuring the absorbance at $540 \mathrm{~nm}$ with an Elisa microplate reader. Statistical analyzes were performed with the Oneway ANOVA test, using the GraphPad Prism 4.0 software. 


\section{Apoptosis assays}

For apoptosis evaluation, cells were seeded in 12-well plates at density of $5 \times 10^{4}$ cells/well. After 24 hours of incubation under normal culture condition, FBS was removed and cells were incubated further for 144 hours. After that, cells were washed in ice-cold PBS and stained with annexin V for 15 minutes at RT. PI was added $(2 \mathrm{ng} / \mu \mathrm{L})$ prior to the FACS analysis, performed using FACSCanto and FACSDIVa software (BD Biosciences). Statistical analyzes were performed with the One-way ANOVA test, using the GraphPad Prism 4.0 software.

\section{Clonogenic survival assay}

Stably transfected U87MG and T98G cells expressing of the ectopic gene (PIMREG and LUC) or miRNAembedded siRNA sequences (miPIMREG\#1, miPIMREG\#2 and miLUC) were seeded in 6-well plates at density of 500-2500 cells/well and treated with TMZ. The drug was removed and cells were further incubated for 9 days under normal culture conditions. After that period, cells were fixed with $4 \%$ PFA, stained with $0.5 \%$ crystal violet and the number of colonies formed were counted. Statistical analyzes were performed with the One-way ANOVA test, using the GraphPad Prism 4.0 software.

\section{Migration assays}

Migration of U87MG and T98G cells expressing of the ectopic gene (PIMREG and LUC) or miRNAembedded siRNA sequences (miPIMREG\#1, miPIMREG\#2 and miLUC) was assessed by two independent methods.

For the wound healing assay, cells were grown to confluence and wounded by making a scratch with a sterile pipette tip. Images were acquired at 0,72 and 144 hours following the scratch using an inverted bright field microscope. ImageJ v1.38 software was used to calculate the percentage of wound closure determined by reduction of the distance between the wound edges from time 0 set as 100\%. Statistical analyzes were performed with the One-way ANOVA test, using the GraphPad Prism 4.0 software.

Transwell migration experiments were performed in $8 \mu \mathrm{m}$ pore-sized transwell plates (Greiner Bio-One). Cells were seeded above the filter at a density of $3 \times 10^{4}$ cells/well in $200 \mu \mathrm{l}$ of serum-free medium and the lower compartment was filled with and $500 \mu \mathrm{L}$ of $10 \%$ FBS containing medium. After 24 hours, the cells which migrated through the filter and reached the lower compartment were fixed with $4 \%$ PFA and stained with crystal violet $0.1 \%$. The migrated cells were counted using an inverted microscope. Statistical analyzes were performed with the One-way ANOVA test, using the GraphPad Prism 4.0 software.

\section{Declarations}

\section{Acknowledgements}


We thank Dr. Jeremy Andrew Squire for English review and Dr. Sara T. O. Saad for kindly providing the anti-ATR antibodies. This research was supported by the Fundação de Amparo à Pesquisa de São Paulo (FAPESP; 2019/26035-1 to LFA).

\section{Authors' contributions}

L.F.A. and V.V. designed the study; L.F.A. and R.B.S. designed the experiments, analyzed the data, wrote the paper and prepared the figures; R.B.S., C.C. and V.C.A. performed the experiments. All authors contributed to draft the manuscript, read and approved the final manuscript.

\section{Declaration of competing interests}

The authors have nothing to disclose.

\section{Data sharing}

All data relevant for the study are shown in the manuscript or in the supplementary materials. Unprocessed data will be made available upon reasonable request.

\section{References}

1. Ostrom, Q. T. et al. The epidemiology of glioma in adults: A state of the science review. Neuro. Oncol. 16, 896-913 (2014).

2. Louis, D. N. et al. The 2016 World Health Organization Classification of Tumors of the Central Nervous System: a summary. Acta Neuropathol. 131, 803-820 (2016).

3. Ostrom, Q. T., Gittleman, H., Stetson, L., Virk, S. M. \& Barnholtz-Sloan, J. Current Understanding and Treatment of Gliomas. in Cancer Treatment and Research (eds. Raizer, J. \& Parsa, A.) vol. 163114(2015).

4. McNeill, K. A. Epidemiology of Brain Tumors. Neurol. Clin. 34, 981-998 (2016).

5. Van Meir, E. G. et al. Exciting New Advances in Neuro-Oncology. CA. Cancer J. Clin. 60, 166-193 (2010).

6. Ceccarelli, M. et al. Molecular profiling reveals biologically discrete subsets and pathways of progression in diffuse glioma. 164,550-563(2016).

7. Verhaak, R. G. W. et al. Integrated Genomic Analysis Identifies Clinically Relevant Subtypes of Glioblastoma Characterized by Abnormalities in PDGFRA, IDH1, EGFR, and NF1. Cancer Cell. 17, 98110 (2010).

8. Noushmehr, H. et al. Identification of a CpG Island Methylator Phenotype that Defines a Distinct Subgroup of Glioma. Cancer Cell. 17, 510-522 (2010).

9. Archangelo, L. F. et al. The CALM and CALM/AF10 interactor CATS is a marker for proliferation. Mol. Oncol. 2, 356-367 (2008). 
10. Zhao, W. M. et al. RCS1, a substrate of APC/C, controls the metaphase to anaphase transition. Proc. Natl. Acad. Sci. U. S. A. 105, 13415-13420(2008).

11. Archangelo, L. F., Gläsner, J., Krause, A. \& Bohlander, S. K. The novel CALM interactor CATS influences the subcellular localization of the leukemogenic fusion protein CALM/AF10. Oncogene. 25, 40994109 (2006).

12. Barbutti, I. et al. The U2AF homology motif kinase 1 (UHMK1) is upregulated upon hematopoietic cell differentiation. Biochim. Biophys. Acta - Mol. Basis Dis. 1864, 959-966 (2018).

13. Zhang, C. et al. Integrated analysis of expression profiling data identifies three genes in correlation with poor prognosis of triple-negative breast cancer. Int. J. Oncol. 45, 2025-2033 (2014).

14. Hu, S. et al. Transcriptional response profiles of paired tumor-normal samples offer novel perspectives in pan-cancer analysis. Oncotarget. 8, 41334-41347 (2017).

15. Jiang, L. et al. Overexpression of PIMREG promotes breast cancer aggressiveness via constitutive activation of NF-kB signaling. EBioMedicine. 43, 188-200 (2019).

16. Yao, Z. et al. Knockdown of FAM64A suppresses proliferation and migration of breast cancer cells. Breast Cancer. 26, 835-845 (2019).

17. Zhang, J. et al. Up-regulation of FAM64A promotes epithelial-to-mesenchymal transition and enhances stemness features in breast cancer cells. Biochem. Biophys. Res. Commun. 513, 472-478 (2019).

18. Wei, W. et al. Identification of key genes involved in the metastasis of clear cell renal cell carcinoma. Oncol. Lett. 17, 4321-4328 (2019).

19. Yamada, Y. et al. Regulation of antitumor miR-144-5p targets oncogenes: Direct regulation of syndecan-3 and its clinical significance. Cancer Sci. 109, 2919-2936 (2018).

20. Jiao, Y., Fu, Z., Li, Y., Zhang, W. \& Liu, Y. Aberrant FAM64A mRNA expression is an independent predictor of poor survival in pancreatic cancer.PLoS One14, (2019).

21. Li, Z., Zhang, Y., Zhang, Z., Zhao, Z. \& Lv, Q. A four-gene signature predicts the efficacy of paclitaxelbased neoadjuvant therapy in human epidermal growth factor receptor $2-$ negative breast cancer. $J$. Cell. Biochem. 120, 6046-6056 (2019).

22. Xu, Z. S. et al. FAM64A positively regulates STAT3 activity to promote Th17 differentiation and colitisassociated carcinogenesis. Proc. Natl. Acad. Sci. U. S. A. 116, 10447-10452(2019).

23. Archangelo, L. F. et al. The CATS (FAM64A) protein is a substrate of the Kinase Interacting Stathmin (KIS). Biochim. Biophys. Acta - Mol. Cell Res. 1833, 1269-1279 (2013).

24. Cohen, A. L., Holmen, S. L. \& Colman, H. IDH1 and IDH2 mutations in gliomas. Curr. Neurol. Neurosci. Rep. 13, 1-7 (2013).

25. Bettegowda, C. et al. Mutations in CIC and FUBP1 contribute to human oligodendroglioma. Science (80-.). 333, 1453-1455 (2011).

26. Smith, J. S. et al. Alterations of chromosome arms $1 p$ and $19 q$ as predictors of survival in oligodendrogliomas, astrocytomas, and mixed oligoastrocytomas. J. Clin. Oncol. 18, 636-645 
(2000).

27. Freije, W. A. et al. Gene expression profiling of gliomas strongly predicts survival. Cancer Res. 64, 6503-6510 (2004).

28. Gravendeel, L. A. M. et al. Intrinsic gene expression profiles of gliomas are a better predictor of survival than histology. Cancer Res. 69, 9065-9072 (2009).

29. Phillips, H. S. et al. Molecular subclasses of high-grade glioma predict prognosis, delineate a pattern of disease progression, and resemble stages in neurogenesis. Cancer Cell. 9, 157-173 (2006).

30. Blackford, A. N., Jackson, S. P., ATM, ATR \& and DNA-PK: The Trinity at the Heart of the DNA Damage Response. Mol. Cell. 66, 801-817 (2017).

31. Barbutti, I. et al. CATS (FAM64A) abnormal expression reduces clonogenicity of hematopoietic cells. Oncotarget. 7, 68385-68396 (2016).

32. Matise, M. P. \& Wang, H. Sonic Hedgehog Signaling in the Developing CNS. Where It Has Been and Where It Is Going. Current Topics in Developmental Biology vol. 97(Elsevier Inc., 2011).

33. Santoni, M. et al. Essential Role of Gli Proteins in Glioblastoma Multiforme. Curr. Protein Pept. Sci. 14, 133-140 (2013).

34. Hashimoto, K. et al. Fam64a is a novel cell cycle promoter of hypoxic fetal cardiomyocytes in mice. Sci. Rep. 7, 1-17 (2017).

35. Brązert, M. et al. Human Ovarian Granulosa Cells Isolated during an IVF Procedure Exhibit Differential Expression of Genes Regulating Cell Division and Mitotic Spindle Formation. J. Clin. Med. 8, 2026 (2019).

36. Erasimus, H., Gobin, M., Niclou, S. \& Van Dyck, E. DNA repair mechanisms and their clinical impact in glioblastoma. Mutat. Res. - Rev. Mutat. Res. 769, 19-35 (2016).

37. Anantha, R. W., Vassin, V. M. \& Borowiec, J. A. Sequential and synergistic modification of human RPA stimulates chromosomal DNA repair. J. Biol. Chem. 282, 35910-35923 (2007).

38. Liu, S. et al. Distinct roles for DNA-PK, ATM and ATR in RPA phosphorylation and checkpoint activation in response to replication stress. Nucleic Acids Res. 40, 10780-10794 (2012).

39. Olson, E., Nievera, C. J., Klimovich, V., Fanning, E. \& Wu, X. RPA2 is a direct downstream target for ATR to regulate the S-phase checkpoint. J. Biol. Chem. 281, 39517-39533 (2006).

40. Myers, J. S. \& Cortez, D. Rapid activation of ATR by ionizing radiation requires ATM and Mre11. J. Biol. Chem. 281, 9346-9350 (2006).

41. Stiff, T. et al. ATR-dependent phosphorylation and activation of ATM in response to UV treatment or replication fork stalling. EMBO J. 25, 5775-5782 (2006).

42. Zhang, J., Stevens, F. G., Bradshaw, D., Temozolomide, T. \& M. \&, Mechanisms of Action, Repair and Resistance. Curr. Mol. Pharmacol. 5, 102-114 (2012).

43. Eich, M., Roos, W. P., Nikolova, T. \& Kaina, B. Contribution of ATM and ATR to the resistance of glioblastoma and malignant melanoma cells to the methylating anticancer drug temozolomide. Mol. Cancer Ther. 12, 2529-2540 (2013). 
44. Deng, M., Brägelmann, J., Kryukov, I., Saraiva-Agostinho, N. \& Perner, S. FirebrowseR: An R client to the Broad Institute's Firehose Pipeline. Database. 2017, 1-6 (2017).

45. Cerami et al. The cBio Cancer Genomics Portal: An Open Platform for Exploring Multidimensional Cancer Genomics Data. Cancer Discov. 2, 401-404 (2012).

46. Aguirre-Gamboa, R. et al. SurvExpress: An Online Biomarker Validation Tool and Database for Cancer Gene Expression Data Using Survival Analysis. PLoS One. 8, 1-9 (2013).

47. Huang, D. W., Sherman, B. T. \& Lempicki, R. A. Systematic and integrative analysis of large gene lists using DAVID bioinformatics resources. Nat. Protoc. 4, 44-57 (2009).

48. Paradis, A., Leblanc, D. \& Dumais, N. Optimization of an in vitro human blood-brain barrier model: Application to blood monocyte transmigration assays. MethodsX. 3, 25-34 (2016).

49. Bornkamm, G. W. et al. Stringent doxycycline-dependent control of gene activities using an episomal one-vector system. Nucleic Acids Res. 33, 1-11 (2005).

50. Hölzel, M. et al. Rapid conditional knock-down - Knock-in system for mammalian cells. Nucleic Acids Res.35, (2007).

51. Livak, K. J. \& Schmittgen, T. D. Analysis of relative gene expression data using real-time quantitative PCR and the 2- $\Delta \Delta C T$ method. Methods. 25, 402-408 (2001).

\section{Figures}




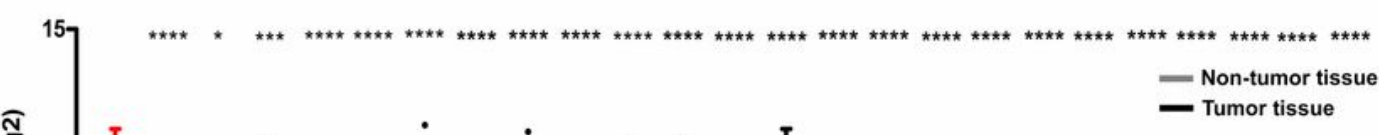

B

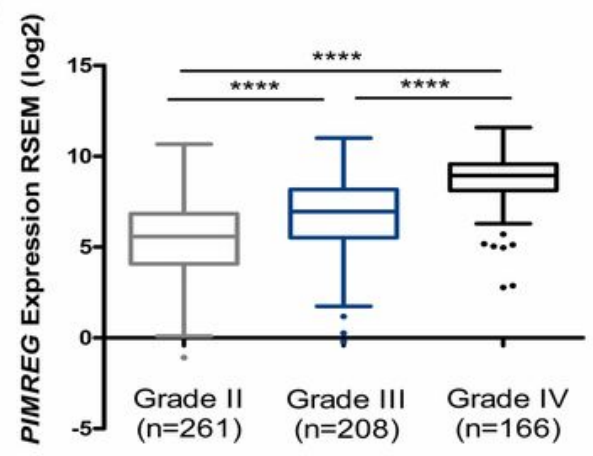

D
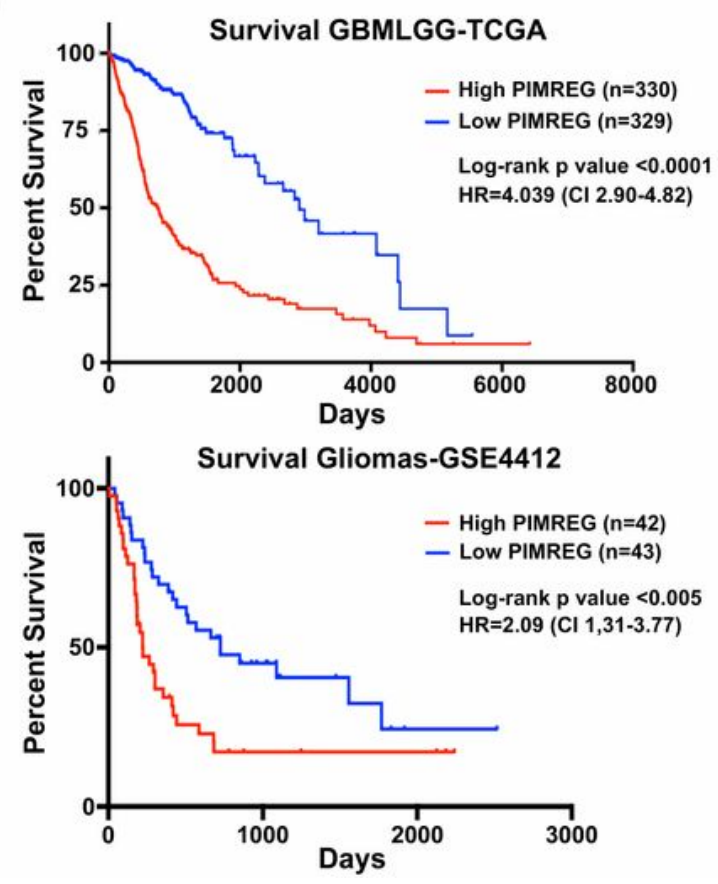

C
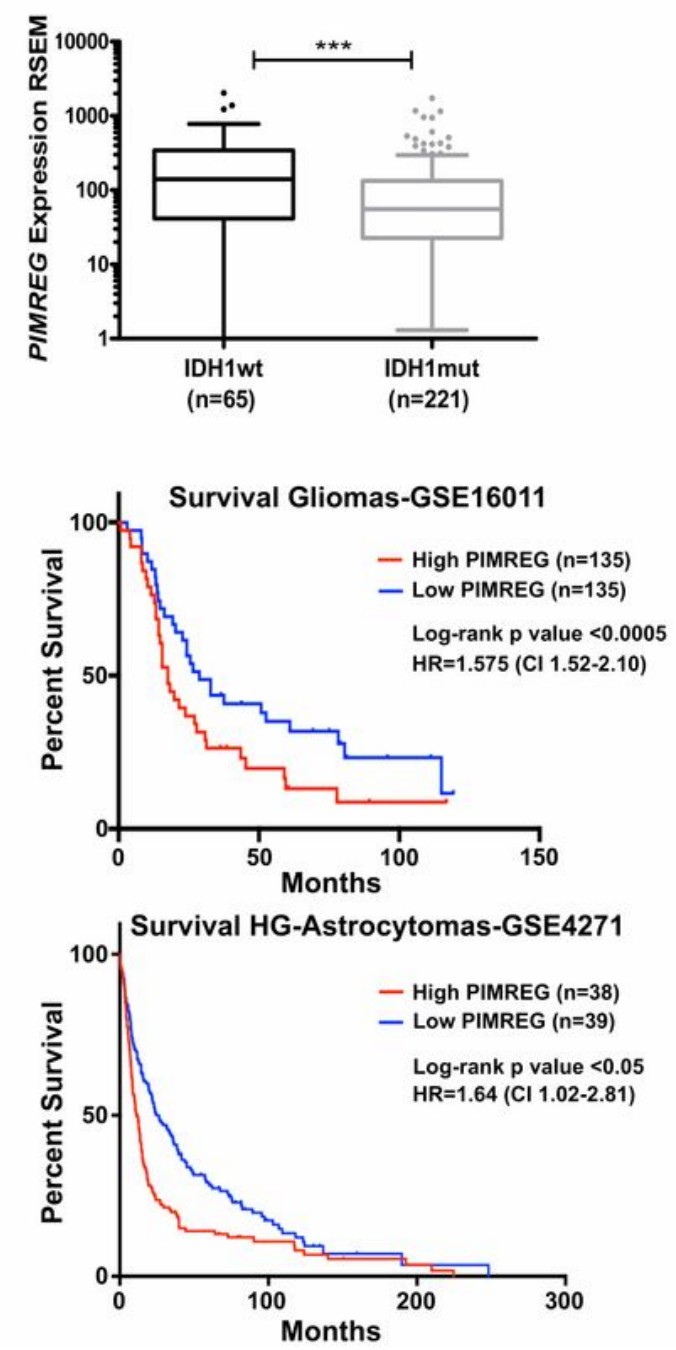

\section{Figure 1}

PIMREG gene expression is enriched in GBM patients, correlates with tumor progression and patient survival. (A) PIMREG expression in paired tumor (shown in black, except for glioblastoma shown in red) and adjacent normal tissue samples (shown in gray) across different TCGA cancer types. Outliers are represented as dots. Tukey's box-and-whisker plot. $\left({ }^{\star}\right) \mathrm{p}$-value $=0.0218 ;\left({ }^{\star \star \star}\right) \mathrm{p}$-value $<0.0008 ;\left({ }^{\star \star \star \star}\right) \mathrm{p}$ value < 0.0001, Kruskal-Wall test for multiple comparisons. GBM: glioblastoma; THYM: thymoma; CESC: 
cervical and endocervical cancers; SARC: sarcoma; LUSC: lung squamous cell carcinoma; COAD: colon adenocarcinoma; BLCA: bladder urothelial carcinoma; READ: rectum adenocarcinoma; UCEC: uterine corpus endometrial carcinoma; HNSC: head and neck squamous cell carcinoma; ESCA: esophageal carcinoma; STES: stomach and esophageal carcinoma; STAD: stomach adenocarcinoma; GBMLGG: GBM and LGG; BRCA: breast invasive carcinoma; CHOL: cholangiocarcinoma; LUAD: lung adenocarcinoma; PAAD: pancreatic adenocarcinoma; KIRC: kidney renal clear cell carcinoma; PRAD: prostate adenocarcinoma; KIRP: kidney renal papillary cell carcinoma; PCPG: pheochromocytoma and paraganglioma; LIHC: liver hepatocellular carcinoma; THCA: thyroid carcinoma; KICH: kidney chromophobe. (B) PIMREG expression levels in the TCGA GBMLGG patients classified according to malignant phenotype. Grade II: astrocytoma, oligodendroglioma and oligoastrocytoma; Grade III:

anaplastic oligoastrocytoma and anaplastic astrocytoma; Grade IV: glioblastoma. ( $\left.{ }^{\star \star \star \star}\right)$ p-value $<0.0001$, Mann-Whitney and Kruskal-Wall test for multiple comparisons. (C) PIMREG expression levels in the TCGA LGG patients classified according to presence of IDH1 mutation. IDH1 wt: IDH wild type phenotype, IDH1 mut: IDH mutated phenotype. ( $\left.{ }^{\star * \star}\right)$ p-value $=0.0006$, Mann-Whitney two-tailed test. (D) Kaplan-Meier survival curves of PIMREG high and PIMREG low groups. The median expression value of PIMREG in the patient data set was used as cutoff. Patients with PIMREG expression above the median were classified as PIMREG high and the patients with PIMREG expression below the median were classified as PIMREG low. Four different cohorts were used for the analysis: GBMLGG-TCGA $(n=660)$, Gliomas-Gravendeel $(n=$ 284), Gliomas-Freije $(n=85)$ and High Grade $(H G)$ Astrocytomas-Phillips $(n=100)$. HR: hazard ratio. Cl: confidence interval. Log-rank test.
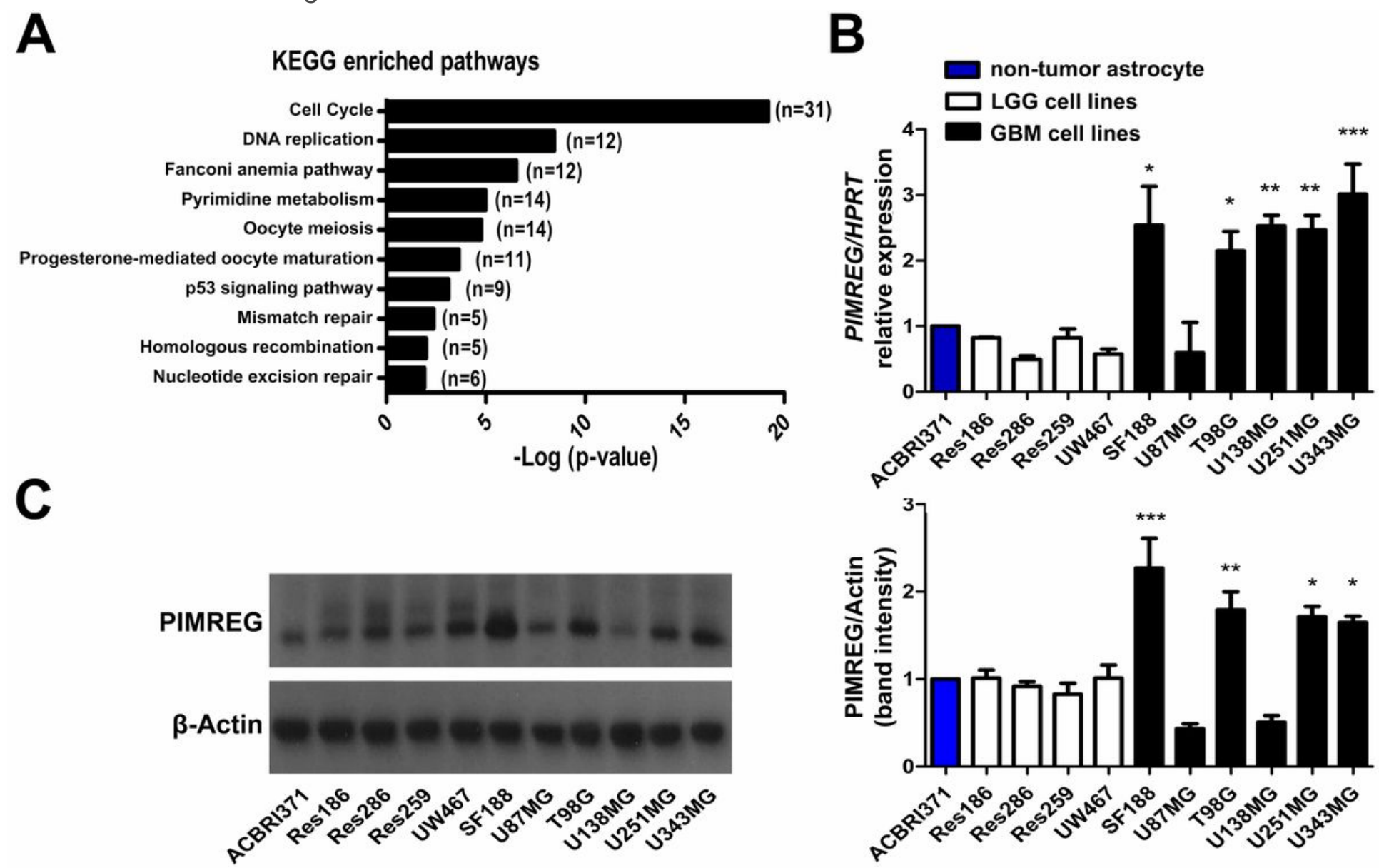


\section{Figure 2}

Pathway enrichment analysis and PIMREG expression in glioblastoma cells. (A) Pathway enrichment analysis (KEEG) of genes whose expression covariates with PIMREG in patient samples (TCGA provisional GBM $n=604$ ). Spearman's correlation coefficient $\geq 0.51$. The number of PIMREG correlated genes in each of the 10 most significant enriched pathways is depicted in brackets. (B) PIMREG mRNA relative expression normalized by HPRT. The non-tumor astrocyte ACBRI371 cells were used as reference for relative expression calculations. LGG: low grade glioma; GBM glioblastoma. (C) Western blot analysis of glioma cells total extracts. Membrane was blotted with PIMREG and $\beta$-Actin antibodies. A representative blot is shown (left panel). The bar graphs represent densitometric measurement of the bands normalized by $\beta$-Actin and relative to control (ACBRI371) set as 1 (right panel). Results are shown as mean \pm SD (standard deviation) of three independent experiment. $\left.{ }^{\star}\right) p$-value $<0.05$, $\left(^{* \star}\right) p$-value $<$ 0.001 , and $\left(^{* \star *}\right)$ p-value $<0.0001$, One-way ANOVA test. 

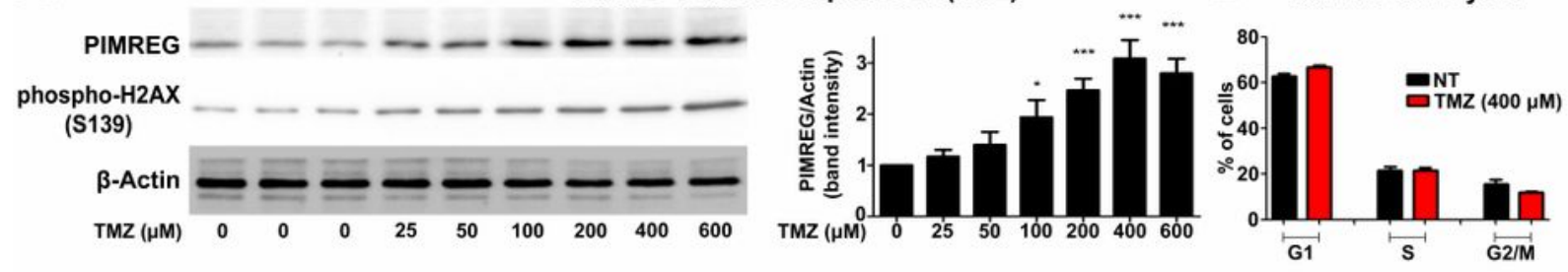

T98G - PIMREG expression (TMZ)

PIMREG

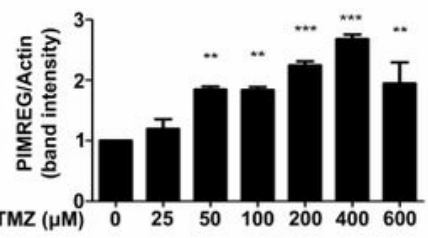

T98G Cell Cycle

phospho-H2AX

(S139)

$\beta$-Actin

$\begin{array}{llllllllll}\operatorname{TMZ}(\mu \mathrm{M}) & 0 & 0 & 0 & 25 & 50 & 100 & 200 & 400 & 600\end{array}$

$\operatorname{TMZ}(\mu \mathrm{M}) \quad 0 \quad 25 \quad 50 \quad 100 \quad 200400 \quad 600$

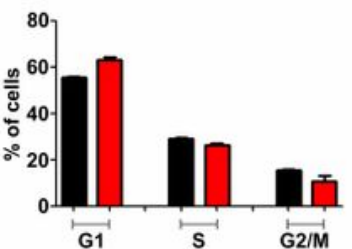

C

U87MG - PIMREG expression (IR)

D U87MG Cell Cycle
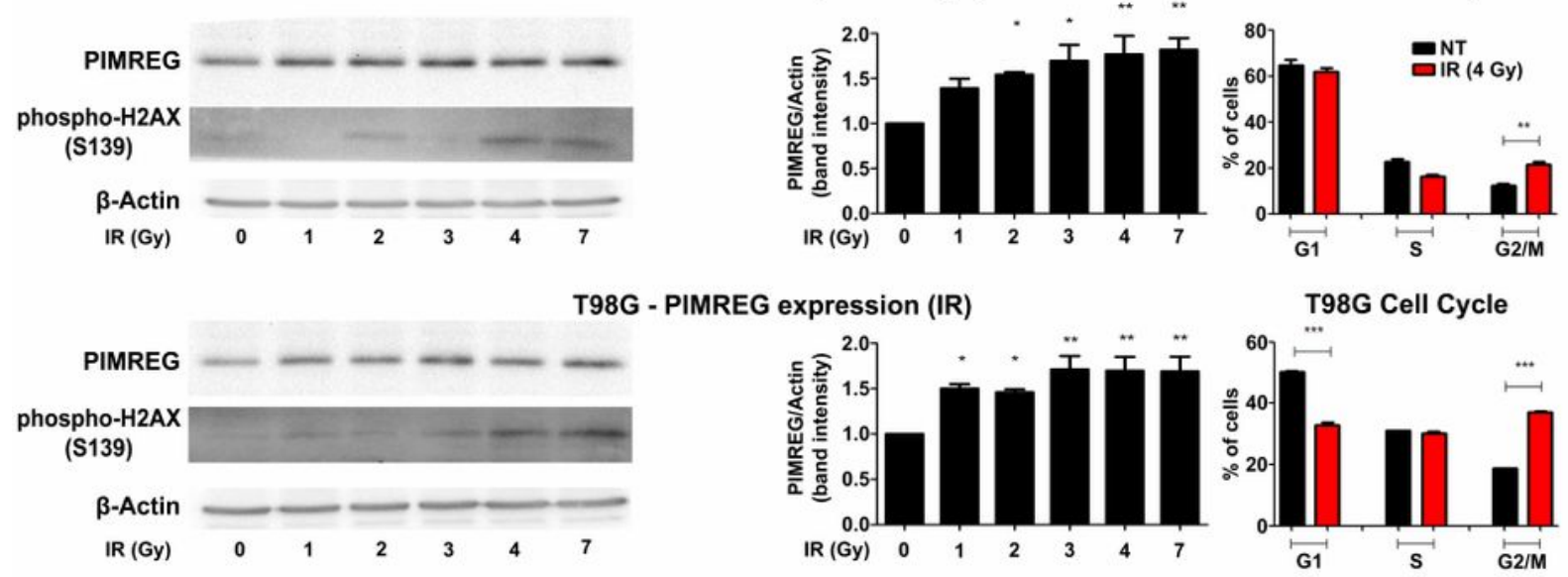

T98G - PIMREG expression (IR)

T98G Cell Cycle
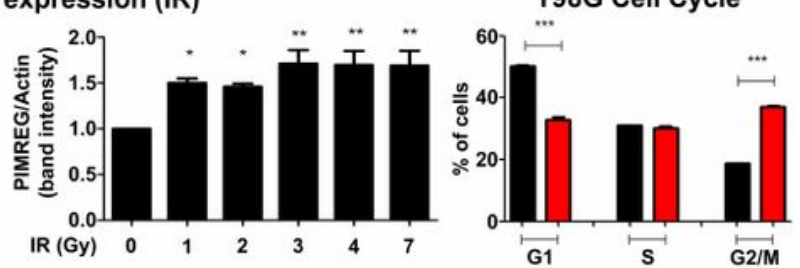

E

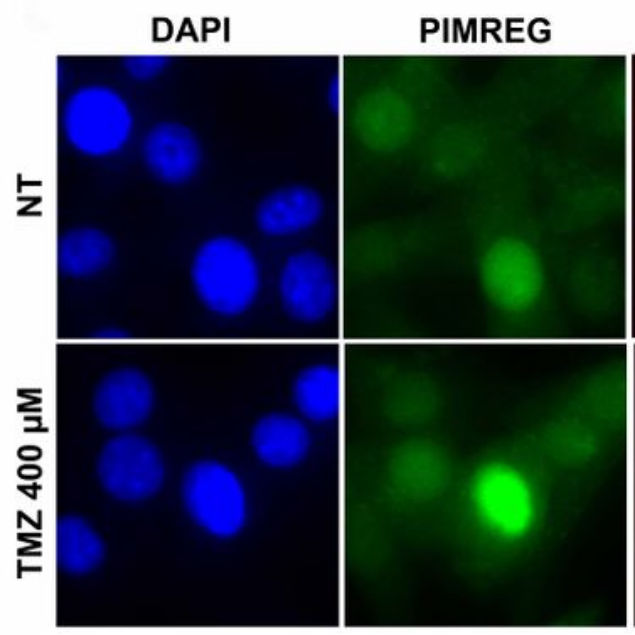

Cyclin A
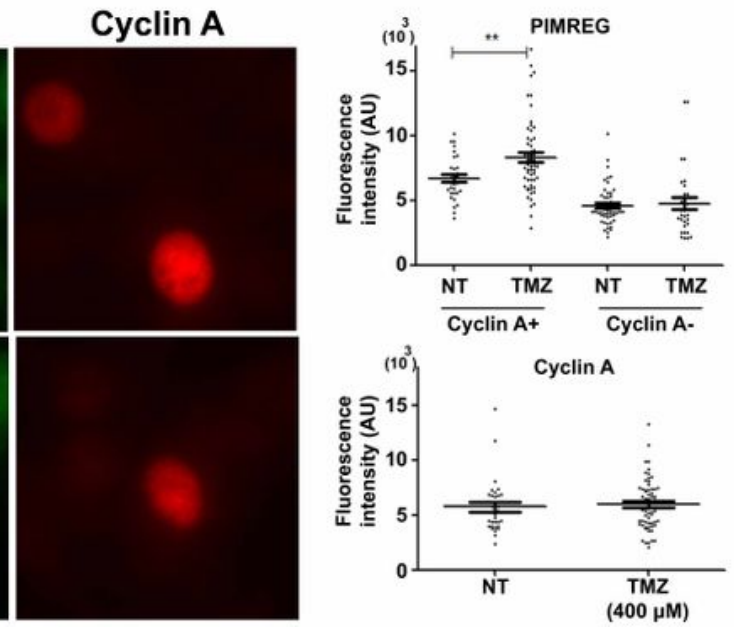

Figure 3

PIMREG expression is induced upon TMZ and IR treatment of GBM cells. $(A ; C)$ Western blot analysis of protein extracts from U87MG and T98G cells treated with TMZ for 24h (A) or 24h after exposure to IR (C). The TMZ concentration and IR dose used are indicated. Membranes were blotted with PIMREG, phospho$\mathrm{H} 2 \mathrm{AX}$ (S139) and $\beta$-Actin antibodies. Representative blots are shown (left panel). The bar graphs represent densitometric measurement of the bands normalized by $\beta$-Actin and relative to untreated 
control cells set as 1 (right panel). Results are shown as mean \pm SD of three independent experiment. One-way ANOVA test. (B;D) Cell cycle analysis of U87MG and T98G cells treated for $24 \mathrm{~h}$ with TMZ (400 $\mu \mathrm{M}$ ) or $24 \mathrm{~h}$ after exposure to ionizing radiation (4 Gy). DNA content was analyzed by flow cytometer. Results are shown as mean \pm SD of three independent experiments. Student's t-test. (e) Nuclear expression of PIMREG and Cyclin A proteins in U87MG cells treated with TMZ $(400 \mu \mathrm{M})$ for $24 \mathrm{~h}$ and analyzed by immunofluorescence microscopy. Fluorescence intensity was quantified by ImageJ v1.38. Values plotted on the graphs (right panels) represent the PIMREG (upper panel) and Cyclin A (lower panel) fluorescence intensity of TMZ non-treated and treated cells. The fluorescence intensity of PIMREG was compared between cells with Cyclin A positive and negative staining (upper graph). Horizontal lines represent the median. 50 cells were evaluated in each condition. Pictures were acquired with $63 \mathrm{X}$ magnification. Student's t-test. TMZ: temozolomide. IR: ionizing radiation. NT: non-treated. AU: Arbitrary Units. $\left(^{*}\right)$ p-value $<0.05,\left({ }^{* *}\right)$-value $<0.001$, and $\left({ }^{* * *}\right)$ p-value $<0.0001$.

\section{A}

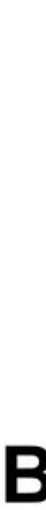

\section{U87MG}

$$
\text { PIMREG }
$$

$\beta$-Actin

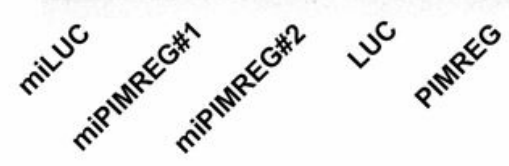

U87MG

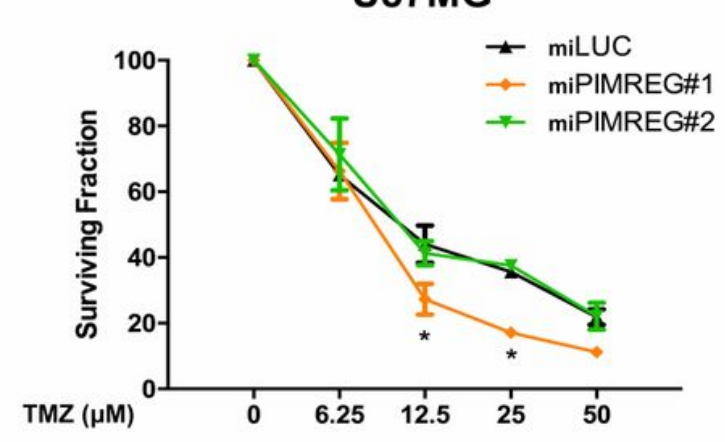

\section{T98G}
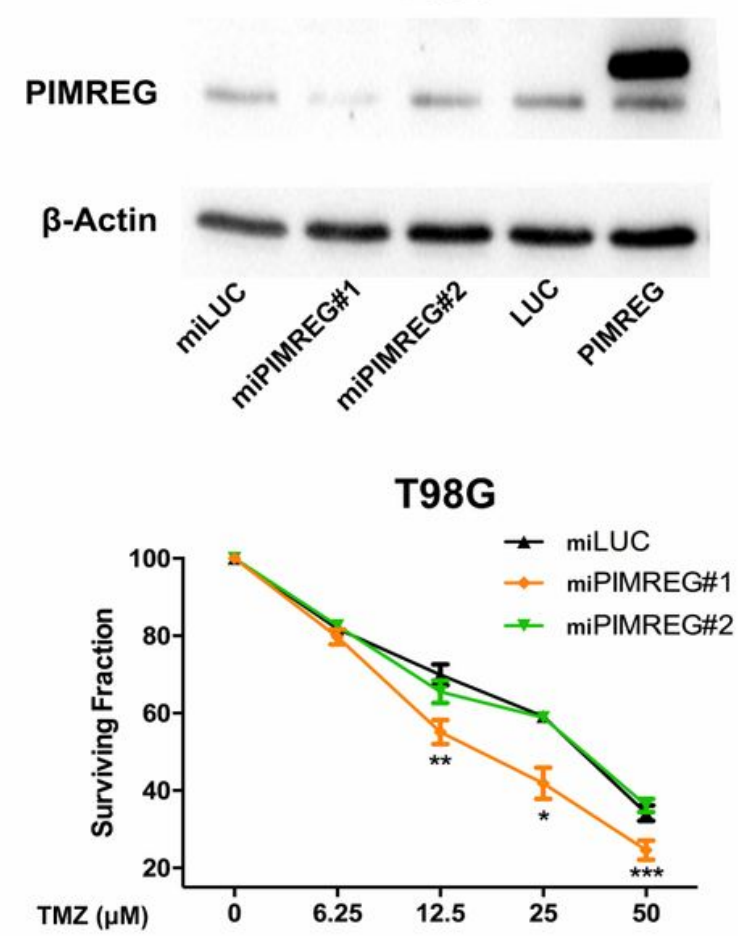

\section{Figure 4}

PIMREG knockdown increases TMZ sensitivity of GBM cells. (A) Western blot analysis of protein extracts from PIMREG overexpressing and depleted U87MG and T98G cells. PIMREG targeting sequence miPIMREG\#1, but not miPIMREG\#2, effectively silenced the gene. Exogenous PIMREG presents higher molecular weight in cellular extracts of T98G cells, possibly because the protein is more phosphorylated in this cell line. The lower band in the same lane shows the endogenous protein. Membranes were blotted with PIMREG and $\beta$-Actin antibodies. (B) Clonogenic survival assay. PIMREG knockdown (miPIMREG\#1, miPIMREG\#2) and control (miLUC) U87MG and T98G cells were treated with different concentration of 
TMZ $(0 \mu \mathrm{M}, 6.25 \mu \mathrm{M}, 12.5 \mu \mathrm{M}, 25 \mu \mathrm{M}$ and $50 \mu \mathrm{M})$ for $24 \mathrm{~h}$ and the number of colonies formed was assessed after 9 days.

\section{A}

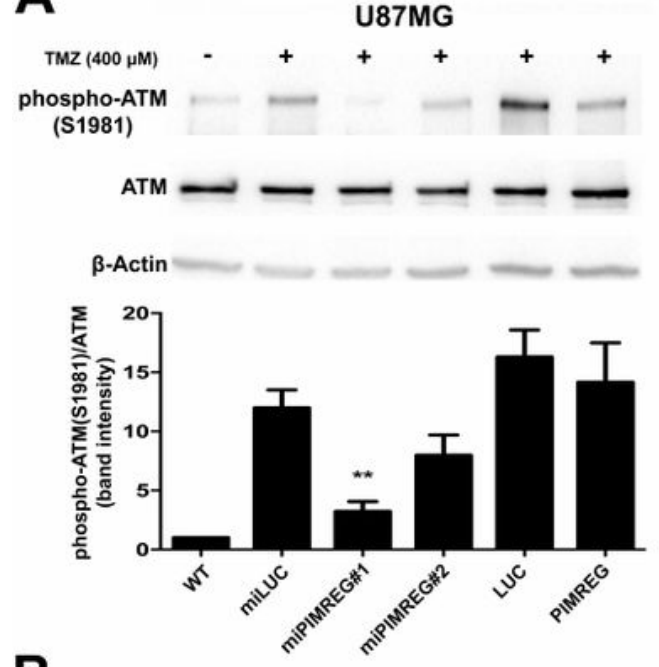

B

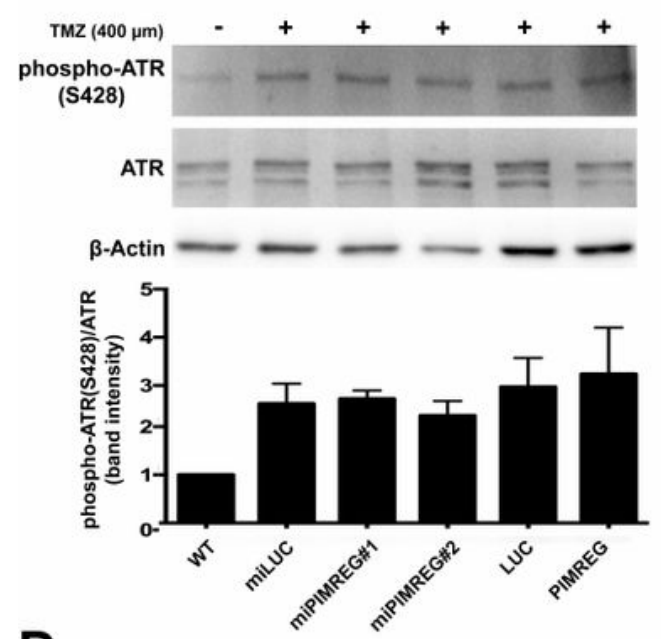

D
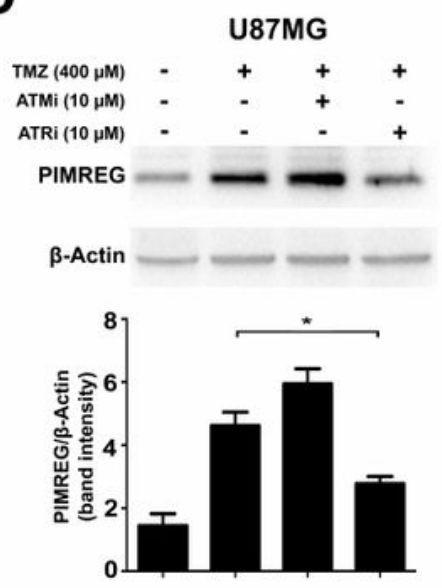

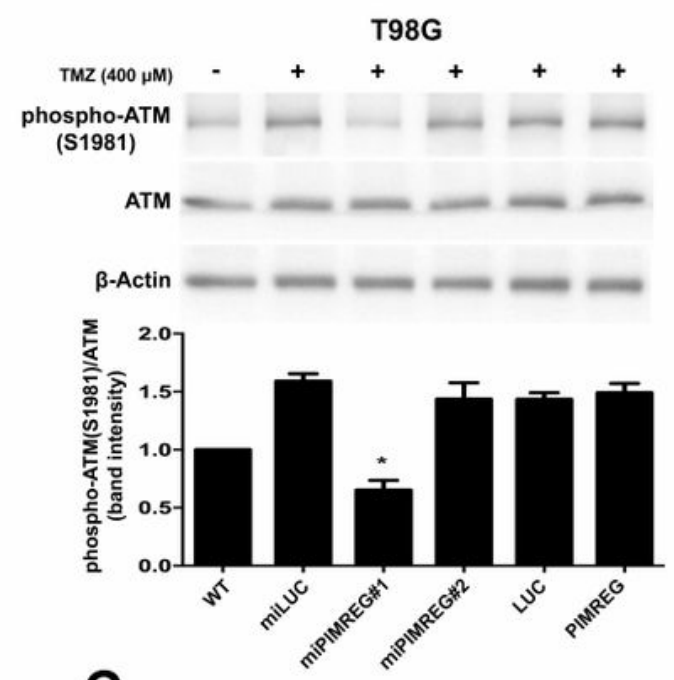

C

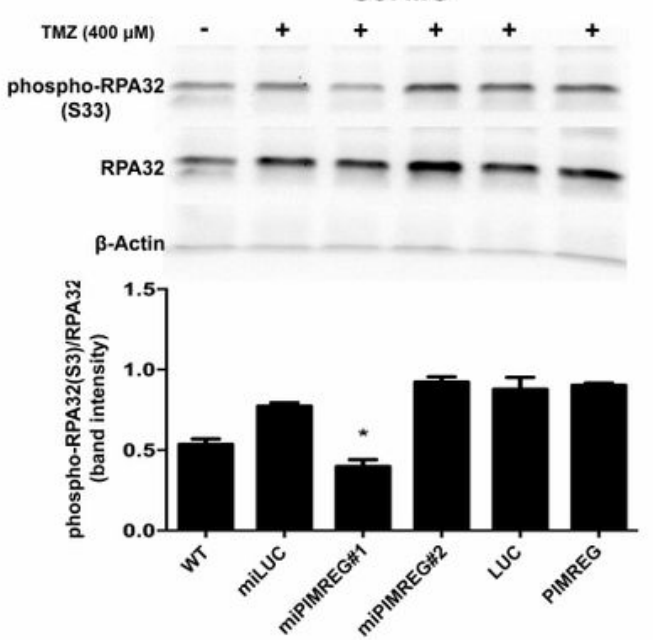

\section{Figure 5}

PIMREG knockdown interferes with the DDR signaling. (A-C) Activity of DNA damage response molecules ATM, ATR and RPA32. Western blot analysis of protein extracts from PIMREG overexpressing and depleted cells treated with TMZ $(400 \mu \mathrm{M})$ for $24 \mathrm{~h}$. Membranes were blotted with the phospho- 
ATM(S1981), total ATM, phospho-ATR(S428), total ATR, phospho-RPA32(S33), total RPA32 and $\beta$-Actin antibodies. Representative blots are shown (upper panels). The bar graphs represent densitometric measurement of the phospho-bands normalized by its respective total protein, relative to untreated wild type control cells (WT) set as 1 (lower panels). (D) Western blot analysis of U87MG cells treated for 3 hours with ATM and ATR inhibitors followed by TMZ. Membrane was blotted with PIMREG and $\beta$-Actin antibodies. Representative blot is shown (upper panel). The bar graph represents densitometric analysis of the bands normalized by $\beta$-Actin and relative to untreated control cells set as 1 (lower panel). Results are shown as mean \pm SD of three independent experiments. $\left({ }^{\star}\right)$ p-value $<0.05$, $\left({ }^{\star *}\right)$ p-value $<0.001$, and $\left.{ }^{(* \star *}\right)$ p-value $<0.0001$, One-way ANOVA test. ATMi: ATM inhibitor. ATRi: ATR inhibitor. 
A

\section{T98G}

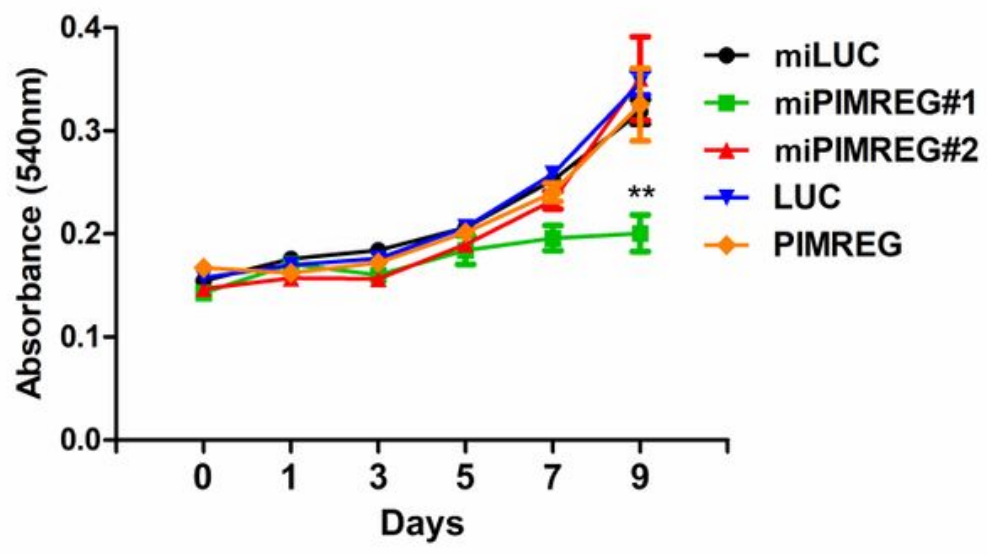

B

\section{Cyclin A}

Cyclin B

$\beta$-Actin
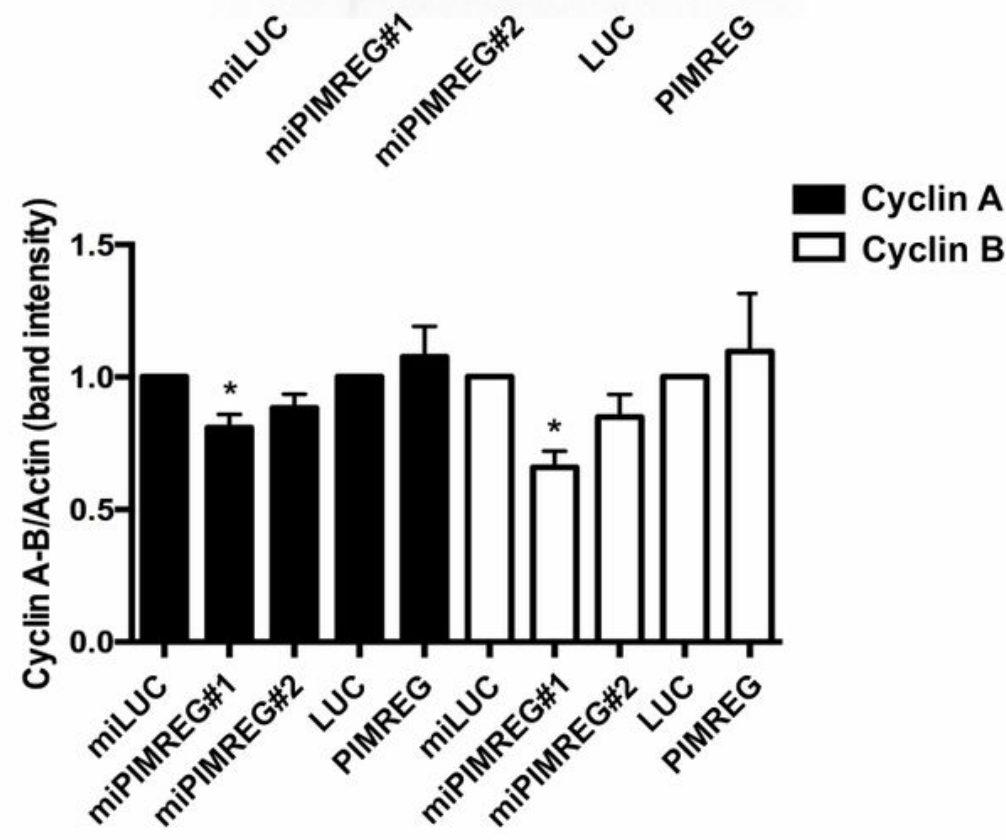

Figure 6

PIMREG knockdown reduces proliferation of T98G cells. (A) Proliferation was assessed by growth curve of PIMREG knockdown (miPIMREG\#1, miPIMREG\#2 and control cells) and PIMREG overexpressing (PIMREG and control) cells. T98G cells cultured in serum depleted medium were assessed for growth on day $0,1,3,5,7$ and 9 of culture. (B) Expression the cell cycle regulator proteins on total cell extracts of PIMREG knockdown (miPIMREG\#1, miPIMREG\#2 and miLUC, used as control) and overexpressing 
(PIMREG and LUC, used as control) cells. Membrane was blotted with the Cyclin A, Cyclin B and $\beta$-Actin antibodies. Representative blot is shown (upper panels). The bar graph represents densitometric measurement of the bands normalized by $\beta$-Actin and relative to miLUC control cells set as 1 (lower panel). Results are shown as mean \pm SD of three independent experiment, $\left(^{\star}\right) p$-value $<0.05$, $\left(^{\star \star}\right) p$-value $<$ 0.001, One-way ANOVA test.

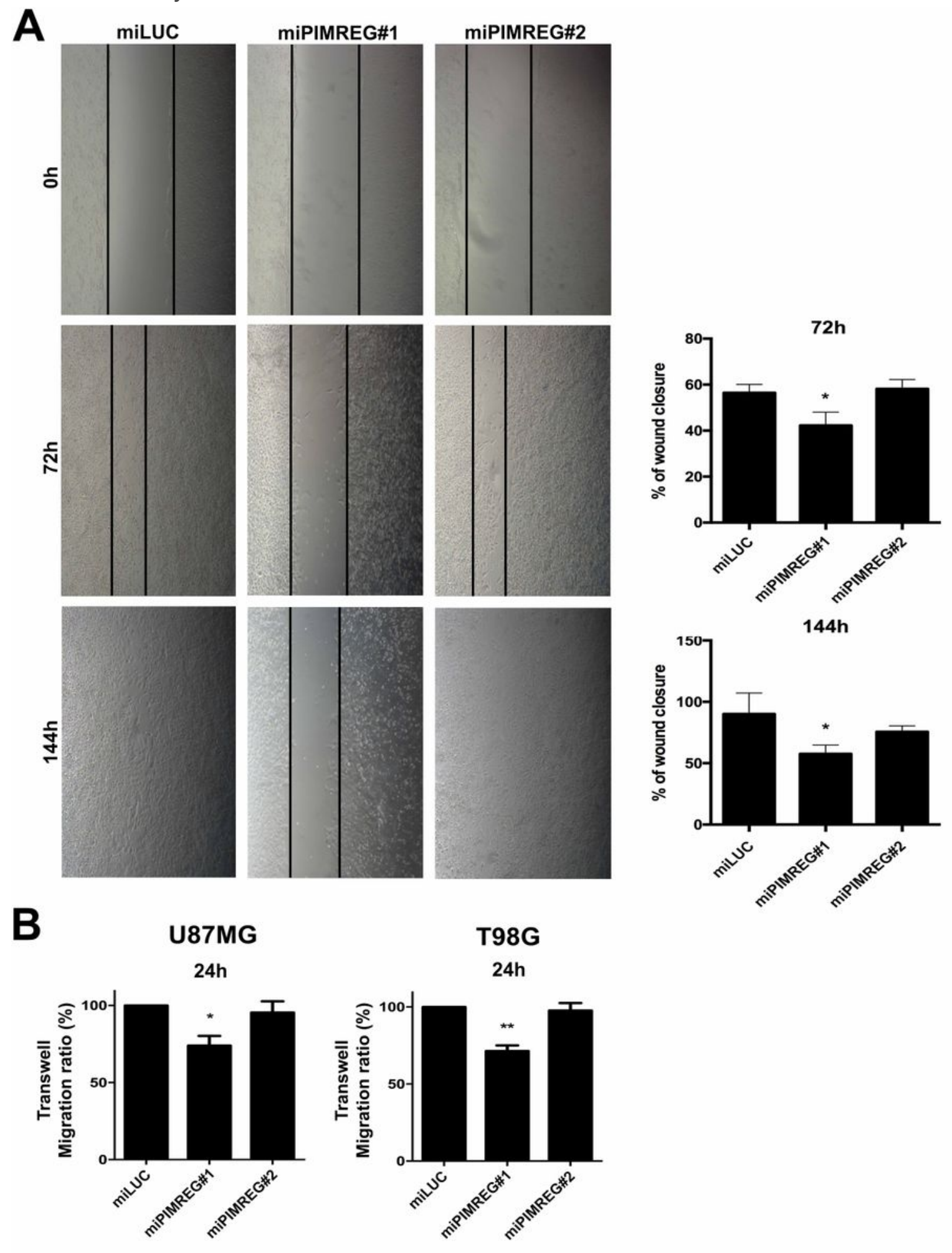

Figure 7 
PIMREG knockdown decreases the migration of GBM cells. (A) Migration was evaluated by wound healing assay. PIMREG knockdown (miPIMREG\#1, miPIMREG\#2) and control (miLUC) T98G cells were grown to confluence and wounded. The pictures were taken in the indicated time points $(0 \mathrm{~h}, 72 \mathrm{~h}$ and 144h). Representative image is shown. The bar graphs represent the percentage of closure, determined by reduction of the distance between the wound edges from time 0 h set as 100\% (right panels). (B) Transwell migration assay. Migration of PIMREG knockdown (miPIMREG\#1, miPIMREG\#2 and miLUC, used as control) U87MG and T98G cells was evaluated by counting the number of cells that passed through the $8 \mu \mathrm{m}$ pore-sized transwell after $24 \mathrm{~h}$ exposure towards FBS-containing media. Results are shown as mean $\pm S D$ of three independent experiments. $\left(^{\star}\right) p$-value $<0.05$, $\left(^{\star *}\right)$ p-value $<0.001$, One-way ANOVA test.

\section{Supplementary Files}

This is a list of supplementary files associated with this preprint. Click to download.

- SupplementarylnformationSR.pdf 